\title{
Agir ou ne pas agir?
}

\section{Werner Bauer}

Dr méd., président de I'ISFM

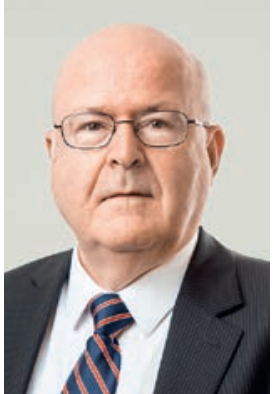

"Chaque fois que nous étions sur le point de créer des communautés, nous avons été réorganisés. Plus tard dans la vie, j'ai appris que nous avions tendance à aborder de nouvelles situations par la réorganisation: c'est une méthode brillante de créer une illusion du progrès pour masquer le désarroi, l'inefficacité et la démoralisation produites."

Titus Petronius Arbiter (env. 14 à 66 ap. J.-C.)

"Take me to the magic of the moment on a glory night, where the children of tomorrow share their dreams with you and me. Take me to the magic of the moment on a glory night, where the children of tomorrow dream away in the wind of change."

Scorpions, Wind of Change (1990)

Poursuivre comme d'habitude ou aller à la recherche du changement là où des évolutions significatives se dessinent? Pour les individus en général et les institutions, la première tendance est d'observer et attendre. Mais il n'est pas rare qu'un conflit ou des questions pressantes déclenchent une forme d'activisme dont se plaignait déjà Pétrone il y a 2000 ans. Les restructurations et les réorganisations peuvent s'avérer impératives ou pertinentes, pourtant elles ne réussissent que si elles s'attaquent au cœur du problème au lieu de ne faire que le masquer.

Qu'est-ce que poursuivre, changer, agir, ne pas agir ont affaire avec un article de ce numéro du Bulletin des médecins suisses sur la répartition du nombre de médecins en formation postgraduée? Une enquête réalisée en 2017 par l'EPFZ à la demande de l'ISFM auprès de tous les médecins-assistants et des responsables d'établissement sur des sujets aujourd'hui particulièrement actuels livre des résultats qui donnent matière à réfléchir sur la nécessité d'agir. Les réponses sont subjectives et se fondent sur l'expérience personnelle mais, dans l'ensemble, elles expriment un avis, celui porté par les établissements de formation en Suisse. Il faut donc le prendre au sérieux. Elles font aussi écho aux réflexions abondamment nourries ces derniers temps par l'administration publique, à savoir une régulation active de la formation des médecins spécialistes assortie d'un raccourcissement de la durée de formation mais aussi l'exigence de demander aux futurs méde- cins de prendre une décision définitive plus tôt qu'aujourd'hui pour choisir une spécialisation.

Les avis concernant l'opportunité d'une régulation ciblée montrent que la question de savoir combien de médecins seront nécessaires demain pour couvrir les besoins à venir en prestations médicales dans les différentes disciplines est légitime. Par contre, une proposition allant dans le sens d'une planification concrète des postes ne dégage aucune majorité. Des différences apparaissent entre les régions et les disciplines: la Suisse francophone et la chirurgie orthopédique sont plus enclines à une régulation que d'autres régions ou spécialisations. En revenant à la question posée dans le titre "Agir ou ne pas agir?», on peut dire qu'il sera pertinent d'agir seulement si nous sommes en mesure de définir le besoin futur de manière suffisamment précise. C'est tout sauf simple, mais ce serait le préalable à tout changement porteur d'une véritable amélioration.

A la question de savoir quel est le moment opportun pour choisir une spécialisation, la majorité adopte une attitude libérale, même si une décision plus précoce permettrait de raccourcir un peu la durée de formation. L'obligation de se déterminer dès les études universitaires ne recueille guère de soutien tandis qu'une première voire une deuxième année d'«apprentissage et d'orientation" est considérée comme pertinente, même si cette expression est désormais marquée d'une connotation négative dans l'administration $\mathrm{pu}$ blique.

Ce qui interpelle et exige un "wind of change», ce sont les réponses par l'affirmative à la question de l'engagement de collaborateurs médecins pour permettre le bon fonctionnement d'une institution sans que les ressources suffisantes soient effectivement disponibles pour leur formation postgraduée. Cela révèle une certaine faiblesse du système libéral actuel, dans lequel les besoins opérationnels priment bien souvent sur les besoins du système de santé pour définir le nombre de postes de diverses disciplines. Ce point doit être pris au sérieux et exige assurément des mesures visant à garantir les ressources financières et l'espace-temps requis et à diminuer les tâches administratives des établissements de formation. Des mesures incitatives allant plus loin seraient pertinentes et acceptables uniquement si elles permettent d'agir et non de réagir. 